\title{
BMJ Open Untargeted antifungal therapy in adult patients with complicated intra- abdominal infection: protocol for a systematic review with meta-analysis
}

Marie Warrer Petersen, ${ }^{1}$ Anders Perner, ${ }^{1}$ Fredrik Sjövall, ${ }^{2}$ Morten Hylander Møller ${ }^{1}$

To cite: Petersen MW, Perner A, Sjövall $\mathrm{F}$, et al. Untargeted antifungal therapy in adult patients with complicated intraabdominal infection: protocol for a systematic review with meta-analysis. BMJ Open 2017;7:e015900. doi:10.1136/ bmjopen-2017-015900

- Prepublication history and additional material for this paper are available online. To view these files please visit the journal online (http://dx.doi. org/10.1136/bmjopen-2017015900).

Received 11 January 2017 Revised 14 March 2017 Accepted 15 March 2017

\section{(a) CrossMark}

${ }^{1}$ Department of Intensive Care, Copenhagen University Hospital Rigshospitalet, Copenhagen, Denmark

${ }^{2}$ Department of Perioperative Medicine, Skane University Hospital, Malmö, Sweden

Correspondence to Marie Warrer Petersen; mariewpetersen@gmail.com

\section{ABSTRACT}

Introduction Intra-abdominal infections are the second most frequent cause of sepsis. In a recent cohort, fungal specimens were found in $51.9 \%$ of all patients with sepsis and peritonitis. Current systematic reviews comparing untargeted antifungal treatment with placebo or no treatment in patients who are critically ill have provided conflicting results, and clinical equipoise exists. Accordingly, we aim to assess patient-important benefits and harms of untargeted antifungal therapy versus placebo or no treatment in adult patients with complicated intraabdominal infection.

Methods and analysis We will conduct a systematic review with meta-analysis and trial sequential analysis of randomised clinical trials assessing any untargeted antifungal therapy compared with placebo or no treatment in adult patients with complicated intra-abdominal infections. The primary outcome is all-cause mortality, and secondary outcomes include adverse events, duration of mechanical ventilation and inotropic support, need for renal replacement therapy, emergence of antibiotic resistance and intensive care unit and hospital lengthof-stay. Conventional meta-analysis, including sensitivity and subgroup analyses, and assessment of the risk of systematic (bias) and random errors will be conducted. The review will be prepared according to the Preferred Reporting Items for Systematic Reviews and MetaAnalyses statement, the Cochrane methodology and the Grading of Recommendations, Assessment, Development and Evaluation.

Ethics and dissemination Ethical approval is not required as this systematic review only includes previously published data. We aim to publish the review in an international peer-reviewed journal.

Trial registration number International Prospective Register of Systematic Reviews registration number: CRD42016053508.

\section{BACKGROUND}

\section{Description of the condition}

Intra-abdominal infections are the second most frequent cause of sepsis in critically ill patients. ${ }^{1}$ Complicated intra-abdominal infection or peritonitis is characterised by inflammation of the peritoneum, most often caused by bacteria or fungi. Primary or

\section{Strengths and limitations of this study}

- The protocol has been prepared according to the Preferred Reporting Items for Systematic Reviews and Meta-Analyses Protocols (PRISMA-P) statement.

- The systematic review will be conducted in accordance with recommendations from the Cochrane Collaboration, the PRISMA statement and the Grading of Recommendations, Assessment, Development and Evaluation guidelines.

- Exclusively patient-important outcome measures, including mortality, adverse events, use of life support and quality of life, will be evaluated.

- The included trials may be heterogeneous.

- Different antifungal treatment strategies (ie, prophylaxis, pre-emptive and empirical therapy) will be assessed.

- New as well as older antifungal agents will be assessed.

spontaneous bacterial peritonitis occurs due to haematogenous dissemination of bacteria or translocation of bacteria through the enteric wall and is managed without surgical intervention. ${ }^{2}$ Secondary peritonitis is the most common form. It develops in relation to disease or injury due to breach of the intestinal wall and requires immediate source control. ${ }^{2}$ Tertiary peritonitis is defined as persistent or reoccurring peritonitis within 48 hours of adequate surgical source control. ${ }^{2}$ All forms are associated with high morbidity and mortality despite administration of relevant antibiotics and/or surgical interventions. ${ }^{1-3}$

In a recent retrospective cohort of critically ill patients with sepsis due to peritonitis, fungal specimens were found in $52 \%$ of all patients. ${ }^{4}$ Candida spp constituted the majority of isolates, in particular C. albicans (60\%), C. glabrata (24\%) and C. tropicalis $(9 \%) .{ }^{4}$ Patients with fungal infection had a significantly higher rate of tertiary peritonitis and a higher overall mortality compared with patients without fungal infection. ${ }^{4}$ 


\section{Description of the intervention}

Untargeted antifungal treatment is defined as any antifungal intervention initiated before definitive microbiological evidence of fungi exists. ${ }^{5-8}$ Currently, three different untargeted treatment strategies have been defined, namely prophylaxis, pre-emptive and empirical therapy ${ }^{5-8}$ Antifungal prophylaxis is used in patients with high risk of developing invasive fungal infections, including critical illness, recent abdominal surgery, haematological malignancy, organ transplantation and treatment with glucocorticoids or broad-spectrum antibiotics. ${ }^{5-9}$ Pre-emptive antifungal treatment is administered in response to direct or indirect microbiological evidence of fungi without clinical suspicion of invasive fungal infection. ${ }^{5-8}$ Lastly, empirical antifungal treatment is used in patients with known risk factors and suspicion of fungal infection. ${ }^{5-8}$ In daily clinical practice, it is often difficult to distinguish the different untargeted antifungal treatment strategies.

\section{How the intervention might work}

Diagnosing fungal infection is challenging, as symptoms and signs are non-specific and mimic bacterial infections. ${ }^{9}$ Also, the time to acquire definite diagnosis takes several days as it is still largely based on cultures. Thus, untargeted therapy strategies appear intuitively attractive.

In a prospective, population-based surveillance study of patients with Candida bloodstream infection, early administration of untargeted antifungal treatment was associated with reduced mortality. ${ }^{10} 11$ Similarly, two previous systematic reviews investigating prophylactic antifungal treatment with fluconazole or ketoconazole in non-neutropenic, critically ill patients demonstrated a reduction in both invasive fungal infection and all-cause mortality compared with placebo or no treatment. ${ }^{12}{ }^{13}$ However, in a recently updated Cochrane review including a total of 2761 non-neutropenic, critically ill adults and children, untargeted antifungal treatment did not significantly reduce mortality (moderate quality of evidence). The results did indicate a reduction in rates of invasive fungal infections (low quality of evidence).$^{5}$ In conclusion, existing evidence have provided conflicting results regarding the use of untargeted antifungal therapy. ${ }^{51213}$

\section{Why it is important to do this review}

Several disadvantages of antifungal treatment exist, including drug interactions, side effects and economical expenses. In addition, resistance is increasing, in particular to fluconazole, highlighting the need for balancing benefits and harms of untargeted antifungal therapy. ${ }^{1}$ Existing systematic reviews and meta-analyses on the matter are confined to patients who are critically ill. It remains to be elucidated if all or certain subgroups of adult patients with complicated intra-abdominal infection would benefit from treatment with untargeted antifungal therapy.

\section{Objectives}

We aim to assess patient-important benefits and harms of untargeted antifungal therapy versus placebo or no treatment in adult patients with complicated intra-abdominal infection.

\section{METHODS}

This protocol has been prepared according to the Preferred Reporting Items for Systematic Reviews and Meta-Analyses Protocols (PRISMA-P) statement. ${ }^{14}$ Refer to online supplementary Appendix for the PRISMA-P checklist. The systematic review will be conducted in accordance with recommendations from the PRISMA statement, ${ }^{15}$ and the quality of evidence will be evaluated using the Grading of Recommendations, Assessment, Development and Evaluation (GRADE) approach. ${ }^{16}$ This protocol has also been registered in the International Prospective Register of Systematic Reviews on the 21 December 2016 (trial registration number CRD42016053508).

\section{Types of studies}

We aim to include randomised clinical trials (RCTs) assessing untargeted antifungal therapy in adult patients with complicated intra-abdominal infections (as defined by the original trials). RCTs regardless of publication status, publication period, blinding and language will be included. Cross-over trials and quasirandomised trials will be excluded.

\section{Types of participants}

We will include trials conducted in adult patients (as defined by the original trials) with complicated intra-abdominal infection. Trials conducted in neutropenic as well as non-neutropenic patients will be included. RCTs conducted in animals, children and healthy subjects will be excluded.

\section{Types of interventions}

The interventions of interest include any type of untargeted antifungal therapy, including azoles, echinocandins, polyenes, allylamines and nucleoside analogues in any dose, timing, formulation and duration. Trials are permitted to have more than one intervention group. The comparators are patients receiving either placebo or no treatment.

\section{Types of outcome measures}

Exclusively patient-important outcome measures will be evaluated. ${ }^{17}$ The primary outcome measure is all-cause short-term mortality ( $\leq 90$ days, including in-ICU (intensive care unit) and in-hospital mortality). Secondary outcomes include (1) long-term mortality ( $>90$ days), (2) adverse events (as defined by the original trials) at longest follow-up, (3) duration of mechanical ventilation, (4) days free of mechanical ventilation, (5) need for renal replacement therapy at longest follow-up, (6) days free of renal replacement therapy, (7) duration of vasopressor/inotropic support, (8) days free of vasopressors/ 
inotropes, (9) emergence of antibiotic resistance at longest follow-up, (10) emergence of fungi not susceptible to given antifungal agent, (11) ICU length-of-stay (LOS), (12) hospital LOS and (13) quality of life (as defined by the original trials) at longest follow-up. If multiple time points are reported, we will use and report the outcome with longest follow-up.

\section{Search methods for identification of studies \\ Electronic searches}

We will systematically search the Cochrane Library (Wiley interface, current issue), Medline (OVID interface, 1946 onwards), Embase (OVID interface, 1980 onwards) and Epistemonikos. Refer to online supplementary Appendix for example of full search strategy performed in Medline.

\section{Searching other resources}

Additionally, we will hand-search reference lists of relevant trials and other systematic reviews of untargeted antifungal therapy. Unpublished trials will be sought identified by performing an equivalent search strategy in other registers (eg, ClinicalTrials.gov, European Clinical Trials Database).

\section{Data collection and analysis \\ Selection of studies}

Two independent authors will screen titles and abstracts of identified trials. Relevant trials will be evaluated in full text for eligibility. Disagreements will be resolved by discussion between authors and finally by consensus among all authors.

\section{Data extraction and management}

Two independent authors will extract data from included trials in duplicate using a standardised data extraction form. Data items abstracted will include trial characteristics, patient characteristics, details of intervention(s) and comparator(s), risk of bias and the predefined patient-important outcome measures. We aim to include data from intention-to-treat analysis rather than per protocol. Disagreements will be resolved by discussion between data extracting authors and finally by consensus among all authors.

\section{Measures of treatment effect}

Dichotomous data will be analysed by calculating the cumulative relative risk (RR) with $95 \%$ confidence interval (CI). For continuous data, we will calculate the mean difference with corresponding standard deviation (SD).

\section{Assessment of risk of bias in included studies}

Two authors will independently assess risk of bias of the included trials in accordance with the recommendations from the Cochrane Collaboration. ${ }^{18}$ The domains reviewed include (1) random sequence generation, (2) allocation concealment, (3) blinding of participants and personnel, (4) blinding of outcome assessors, (5) incomplete outcome data, (6) selective outcome reporting and
(7) other bias, including baseline imbalance, early stopping, bias due to vested financial interest and academic bias. If one or more domains are judged as being high or unclear, we will classify the trial as having an overall high risk of bias.

\section{Assessment of the risk of random errors}

The risk of random errors will be assessed by trial sequential analysis (TSA). ${ }^{19}$ TSA is a sample size calculation (interim analysis) for meta-analyses that widens the CIs in case data are too sparse to draw firm conclusions. ${ }^{19}$

We will apply trial sequential monitoring boundaries according to an information size suggested by the trials with low risk of bias and an a priori $20 \%$ RR reduction, alpha $5 \%$, beta $90 \%$ and a control event proportion as per the control arm. ${ }^{19}$

\section{Dealing with missing data}

Authors will be contacted for additional data if relevant.

\section{Assessment of heterogeneity}

We will calculate inconsistency factor $\left(\mathrm{I}^{2}\right)$ and diversity factor $\left(\mathrm{D}^{2}\right)$ to quantify heterogeneity among included trials. We will use both fixed effects and random effects modelling and report the most conservative estimate.

\section{Assessment of small trial bias}

We will assess the risk of small trial bias (publication bias) if 10 or more trials are included by visually examining the funnel plots for asymmetry. ${ }^{20}$

\section{Data synthesis}

Review Manager (RevMan V.5.3) will be used as statistical software to conduct the meta-analyses, including subgroup analyses (summary estimates). For the TSAs we will use the TSA software available from Copenhagen Trial Unit, Denmark.

\section{Subgroup analysis}

We will perform the following subgroup analyses by comparing estimates of the pooled intervention effect in each subgroup, if two of more trials exist:

- Overall low risk of bias versus overall high risk of bias. Hypothesised direction of subgroup effect: increased intervention effect in trials with high risk of bias.

- Prophylactic versus pre-emptive versus empirical treatment strategies. Hypothesised direction of subgroup effect: increased intervention effect in trials assessing empirical antifungal treatment.

- Non-ICU trials versus ICU trials. Hypothesised direction of subgroup effect: increased intervention effect in trials conducted in the ICU.

- Trials published before the year 2000 versus in and after the year 2000. Hypothesised direction of subgroup: increased intervention effect in trials published before the year 2000.

- Patients with primary versus secondary versus tertiary peritonitis. Hypothesised direction of 
subgroup effect: increased intervention effect in trials conducted in patients with tertiary peritonitis.

- Patients with versus without septic shock. Hypothesised direction of subgroup effect: increased intervention effect in trials conducted in patients with septic shock.

- Median/mean Mannheim Peritonitis Index (MPI) score $>25$ versus trials with a median/mean baseline MPI score $\leq 25$. Hypothesised direction of subgroup effect: increased intervention effect in trials conducted on patients with a MPI score $>25$.

- Patients with neutropenia versus patients without neutropenia. Hypothesised direction of subgroup effect: increased intervention effect in trials conducted in patients who are neutropenic.

We will use $\chi^{2}$ test to assess statistical heterogeneity between studies (test of interaction) with a p value of 0.10 considered statistically significant.

\section{Sensitivity analysis}

In the zero event trials, empirical continuity correction will be applied. ${ }^{21}$

\section{Summary of findings}

The quality of evidence for each outcome will be assessed according to GRADE. ${ }^{16}$ The domains assessed include risk of bias, inconsistency, indirectness, imprecision and publication bias. ${ }^{16}$

Contributors MWP, FS, AP and MH drafted the protocol. MH and FS developed the idea for the review and are the guarantors of the review.

Funding This research project received no specific grant from any funding agency in the public, commercial or not-for-profit sectors.

Competing interests None declared.

Provenance and peer review Not commissioned; externally peer reviewed.

Open Access This is an Open Access article distributed in accordance with the Creative Commons Attribution Non Commercial (CC BY-NC 4.0) license, which permits others to distribute, remix, adapt, build upon this work non-commercially, and license their derivative works on different terms, provided the original work is properly cited and the use is non-commercial. See: http://creativecommons.org/ licenses/by-nc/4.0/

(c) Article author(s) (or their employer(s) unless otherwise stated in the text of the article) 2017. All rights reserved. No commercial use is permitted unless otherwise expressly granted.
REFERENCES

1. De Waele JJ. Abdominal sepsis. Curr Infect Dis Rep 2016;18:23.

2. Mishra SP, Tiwary SK, Mishra M, et al. An introduction of tertiary peritonitis. J Emerg Trauma Shock 2014;7:121-3.

3. Montravers P, Blot S, Dimopoulos G, et al. Therapeutic management of peritonitis: a comprehensive guide for intensivists. Intensive Care Med 2016;42:1234-47.

4. Lichtenstern C, Herold C, Mieth M, et al. Relevance of Candida and other mycoses for morbidity and mortality in severe sepsis and septic shock due to peritonitis. Mycoses 2015;58:399-407.

5. Cortegiani A, Russotto V, Maggiore A, et al. Antifungal agents for preventing fungal infections in non-neutropenic critically ill patients. Cochrane Database Syst Rev 2016;1:CD004920.

6. Cortegiani A, Russotto V, Raineri SM, et al. The paradox of the evidence about invasive fungal infection prevention. Crit Care 2016;20:114

7. Cortegiani A, Russotto V, Raineri SM, et al. Should we administer antifungal drugs before the diagnosis of invasive fungal infection in non-neutropenic critically ill patients? Turk J Anaesthesiol Reanim 2016:44:276-8

8. Cortegiani A, Russotto V, Raineri SM, et al. Uncertainty about the evidence on untargeted antifungal treatment. Eur J Intern Med 2017;37:e18-e19.

9. Kullberg BJ, Arendrup MC. Invasive candidiasis. N Engl J Med 2016;374:794-5.

10. Puig-Asensio M, Padilla B, Garnacho-Montero J, et al. Epidemiology and predictive factors for early and late mortality in Candida bloodstream infections: a population-based surveillance in Spain Clin Microbiol Infect 2014;20:0245-54.

11. Bassetti M, Righi E, Ansaldi F, et al. A multicenter study of septic shock due to candidemia: outcomes and predictors of mortality. Intensive Care Med 2014;40:839-45.

12. Cruciani M, de Lalla F, Mengoli C. Prophylaxis of Candida infections in adult trauma and surgical intensive care patients: a systematic review and meta-analysis. Intensive Care Med 2005;31:1479-87.

13. Playford EG, Webster AC, Sorrell TC, et al. Antifungal agents for preventing fungal infections in non-neutropenic critically ill and surgical patients: systematic review and meta-analysis of randomized clinical trials. J Antimicrob Chemother 2006;57:628-38.

14. Shamseer L, Moher D, Clarke M, et al. Preferred reporting items for systematic review and meta-analysis protocols (PRISMA-P) 2015: elaboration and explanation. BMJ 2015;349:g7647.

15. Liberati A, Altman DG, Tetzlaff J, et al. The PRISMA statement for reporting systematic reviews and meta-analyses of studies that evaluate health care interventions: explanation and elaboration. $J$ Clin Epidemiol 2009;62:e1-34.

16. Atkins $D$, Best $D$, Briss PA, et al. Grading quality of evidence and strength of recommendations. BMJ 2004;328:1490.

17. Gaudry S, Messika J, Ricard JD, et al. Patient-important outcomes in randomized controlled trials in critically ill patients: a systematic review. Ann Intensive Care 2017;7:28.

18. The Cochrane Collaboration. Cochrane Handbook for Systematic Reviews of Interventions Version 5.1.0. 2011. http://handbook. cochrane.org.

19. Wetterslev J, Thorlund K, Brok J, et al. Trial sequential analysis may establish when firm evidence is reached in cumulative meta-analysis. $J$ Clin Epidemiol 2008;61:64-75.

20. Sterne JA, Sutton AJ, loannidis JP, et al. Recommendations for examining and interpreting funnel plot asymmetry in meta-analyses of randomised controlled trials. BMJ 2011;343:d4002.

21. Sweeting MJ, Sutton AJ, Lambert PC. What to add to nothing? Use and avoidance of continuity corrections in meta-analysis of sparse data. Stat Med 2004;23:1351-75. 